Revista Iberoamericana

de las Ciencias Sociales y

Humanísticas

ISSN: $2395-7972$

https://doi.org/10.23913/ricsh.v9i17.194

Artículos Científicos

\title{
De viva voz: estudio fenomenológico con agresores primarios de violencia de pareja o conyugal
}

In a Lively Voice: Phenomenological Study with Primary Aggressors of Partner or Spousal Violence

Voz ao vivo: estudo fenomenológico com agressores primários de violência por parceiro ou cônjuge

Elvira Ivone González Jaimes Universidad Autónoma del Estado de México, México ivonegj@hotmail.com http://orcid.org/0000-0002-5328-5586

Ana María Oviedo Zuñiga Universidad Autónoma del Estado de México, México amoviedoz@uaemex.mx https://orcid.org/0000-0002-7597-5174

María Guadalupe Miguel Silva Universidad Autónoma del Estado de México, México mis2529@yahoo.com.mx https://orcid.org/0000-0002-3903-7957

Alejandro Mendieta Vargas Universidad Autónoma del Estado de México, México amendietav@uaemex.mx https://orcid.org/0000-0002-2688-0873 


\section{Revista Iberoamericana \\ de las Ciencias Sociales y \\ Humanísticas}

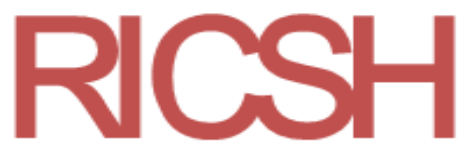

ISSN: 2395 - 7972

\section{Resumen}

La violencia de género en México es alta. De acuerdo con el Instituto Nacional de Estadística y Geografía [Inegi] (22 de noviembre del 2018), 19.1 millones de mujeres adultas mayores de 15 años han enfrentado violencia conyugal o de pareja. La agresión se genera frecuentemente en escenarios privados: por lo vulnerable que se encuentra la mujer ahí. A sabiendas de que el evento de intimidad y agresión es difícil de investigar, en este estudio se entrevistó a 32 participantes (agresores primarios de la pareja) que requerían ser evaluados psicológicamente en los Centros de Atención de la Casa de la Mujer de los municipios de Atizapán y Ecatepec, Estado de México, México. El objetivo fue conocer las causas a través de la propia voz de los agresores primarios, y así obtener una visión objetiva y amplia de los disparadores que producen el fenómeno social de violencia conyugal. Para ello se recurrió a dos tipos de entrevista. La primera fue cerrada y bajo la guía del Manual de Diagnóstico y Estadístico de Trastornos Mentales [DSM-5, por sus siglas en inglés] (American Psychiatric Association [APA], 2013), con el diagnóstico "Relación conflictiva con el cónyuge o la pareja"; y la segunda fue una entrevista semiestructurada para maltratadores de género. El método utilizado fue el análisis de datos de tipo mixto (cuantitativo y cualitativo) para triangular información y entender el qué y el cómo de la agresión de género. Como parte de los resultados, se encontró que la concepción devaluada de la mujer se ve reflejada en el rechazo constante a las conductas positivas de la pareja ( $84 \%$ ), las conductas de la pareja para obtener un valor y ejercer derechos desencadenan en conflictos difíciles de resolver (72\%), porque el agresor está en la idea de conservar su lugar jerárquico de poder y de protección social al ser débil (física y mentalmente), por lo que reconoce la asunción de responsabilidad (66 \%). Esta ambivalencia entre deseo y acción, "querer y no poder", produce tristeza, apatía o rabia crónicas hacia la pareja. Por lo que se recomienda que el agresor primario de la pareja ingrese a pláticas o psicoterapia, donde obtenga justicia terapéutica para que reaprenda cómo relacionarse con su pareja o cónyuge y finalmente cambie sus ideas y rompa con el estereotipo social de machismo.

Palabras claves: agresividad, ciencias sociales, violencia conyugal. 


\section{Revista Iberoamericana \\ de las Ciencias Sociales y \\ Humanísticas}

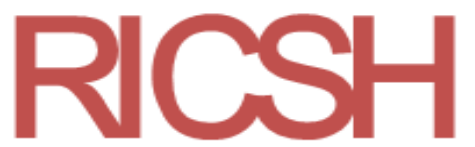

ISSN: $2395-7972$

\section{Abstract}

Gender violence in Mexico is high. According to the Instituto Nacional de Estadística y Geografía [Inegi] (November 22, 2018), 19.1 million adult women over age 15 have faced conjugal o couple violence. Aggression is frequently in private settings because of how vulnerable women are there. Knowing that the intimacy and aggression event is difficult to investigate, 32 participants (primary aggressors of the couple) who required to be evaluated psychologically in the care centers of the Casa de la Mujer in the municipalities of Atizapán and Ecatepec, Estado de México, Mexico, were interviewed. The objective was to obtain an objective and broad vision of the social phenomenon of conjugal violence with the loud voice of the primary aggressors. The objective was to know the causes through the voice of the primary aggressors, and thus obtain an objective and broad vision of the triggers that produce the social phenomenon of conjugal violence. For this, two types of interviews were used. The first one was closed and under the guidance of the Diagnostic and Statistical Manual of Mental Disorders [DSM-5] (American Psychiatric Association, 2013), with the diagnosis "Conflictive relationship with spouse or partner"; and the second was a semistructured interview for gender abusers. The method used was the analysis of mixed-type data (quantitative and qualitative) to triangulate information and understand the what and how of gender aggression. As part of the results, it was found that the devalued conception of women is reflected in the constant rejection of the positive behaviors of the couple (84\%), the couple's behaviors to obtain value and exercise rights trigger in difficult conflicts to resolve (72\%), because the aggressor is in the idea of preserving his hierarchical place of power and social protection, so he recognizes the assumption of responsibility (66\%). This ambivalence between desire and action, "wanting and not being able", produces chronic sadness, apathy or anger towards the couple. Therefore, it is recommended that the couple's primary aggressor enter into talks or psychotherapy, where he obtains therapeutic justice so that he can re-learn how to interact with his partner or spouse and finally change his ideas and break with the social stereotype of machismo.

Keywords: aggression, social sciences, spousal violence. 


\section{Revista Iberoamericana \\ de las Ciencias Sociales y \\ Humanísticas}

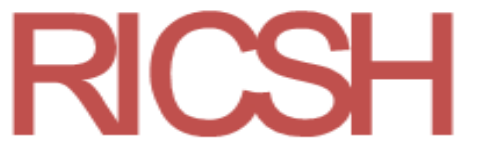

ISSN: $2395-7972$

\section{Resumo}

A violência de gênero no México é alta. De acordo com o Instituto Nacional de Estatística e Geografia [Inegi] (22 de novembro de 2018), 19,1 milhões de mulheres adultas com mais de 15 anos enfrentaram violência conjugal ou conjugal. A agressão é frequentemente gerada em ambientes privados: por causa da vulnerabilidade das mulheres. Sabendo que o evento de intimidade e agressão é difícil de investigar, neste estudo foram entrevistados 32 participantes (agressores primários do casal) que precisavam ser avaliados psicologicamente nos Centros de Atendimento da Casa das Mulheres dos municípios de Atizapán e Ecatepec, Estado do México, México. O objetivo era conhecer as causas pela voz dos agressores primários e, assim, obter uma visão ampla e objetiva dos gatilhos que produzem o fenômeno social da violência conjugal. Para isso, foram utilizados dois tipos de entrevistas. O primeiro foi encerrado e sob a orientação do Manual Diagnóstico e Estatístico de Transtornos Mentais [DSM-5] (Associação Americana de Psiquiatria [APA], 2013), com o diagnóstico "Relação conflitante com o cônjuge ou casal "; e o segundo foi uma entrevista semiestruturada para abusadores de gênero. O método utilizado foi a análise de dados do tipo misto (quantitativa e qualitativa) para triangular informações e entender o que e como da agressão de gênero. Como parte dos resultados, verificou-se que a concepção desvalorizada da mulher se reflete na constante rejeição do comportamento positivo do casal (84\%), os comportamentos do casal para obter valor e exercer direitos desencadeiam conflitos difíceis resolver (72\%), porque o agressor tem a idéia de preservar seu lugar hierárquico de poder e proteção social por ser fraco (físico e mentalmente), e assim reconhece a assunção de responsabilidade (66\%). Essa ambivalência entre desejo e ação, "querer e não poder", produz tristeza crônica, apatia ou raiva em relação ao casal. Portanto, recomenda-se que o agressor primário do casal entre em conversas ou psicoterapia, onde obtém justiça terapêutica para que ele possa reaprender a interagir com seu parceiro ou cônjuge e, finalmente, mudar suas idéias e romper com o estereótipo social do machismo.

Palavras-chave: agressividade, ciências sociais, violência conjugal.

Fecha Recepción: Agosto 2019

Fecha Aceptación: Diciembre 2019 


\section{Revista Iberoamericana \\ de las Ciencias Sociales y \\ Humanísticas}

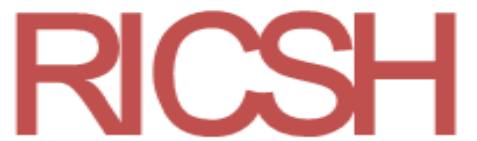

ISSN: $2395-7972$

\section{Introducción}

Esta es una investigación mixta cuantitativa y cualitativa para conocer de forma integral el fenómeno social de la violencia de género desde la perspectiva de los actores (agresores primarios de violencia de género) y entender cómo se desarrolla la violencia en la relación de pareja desde el relato del agresor.

El análisis cuantitativo de tipo descriptivo fue realizado realizado en tres etapas: 1) las características socioeconómicas de la muestra; 2) la frecuencia y porcentaje de los principales síntomas del diagnóstico "Relación conflictiva con el cónyuge o la pareja”, perteneciente al Manual de Diagnóstico y Estadístico de Trastornos Mentales (DSM-5, por sus siglas en inglés) de la American Psychiatric Association (2013), y 3) la frecuencia y porcentaje de las características del área psicosocial y episodios de maltrato estipulados en la entrevista semiestructurada para maltratadores de género con respuesta de opción múltiple (Arce y Fariña, manuscrito no publicado).

El análisis cualitativo, de tipo fenomenológico y enfoque etnometodológico, a partir de las grabaciones de las entrevistas, fue realizado para analizar el contenido de los episodio de agresión contra la pareja, expresados desde el relato hecho por victimarios (agresores primarios en la violencia de género), y para entender el fenómeno de la violencia de género de viva voz de los actores, tomando en cuentas los aspectos circunstanciales. El análisis mixto de triangulación de la información arrojará luz sobre el qué y el cómo de este fenómeno social tan frecuente en México.

En México, la mayoría de la violencia de género es propiciada por la pareja. Según el Instituto Nacional de Estadística y Geografía [Inegi] (22 de noviembre del 2018), 19.1 millones de mujeres adultas mayores de 15 años han enfrentado violencia conyugal o de pareja, cifra equivalente a $64.0 \%$ de las encuestas realizadas. Los casos registrados se tratan de violencia severa y muy severa, según dichas estadísticas difundidas por el Inegi (22 de noviembre del 2018) a propósito del Día Internacional de la Eliminación de la Violencia Contra la Mujer (25 de noviembre). Por lo que se considera a la pareja como el agresor primario por el nivel de vulnerabilidad que manifiesta en esa situación la mujer. Asimismo, la pareja ocupa el segundo lugar como causante de feminicidio, según resultados de la Encuesta Nacional sobre la Dinámica de las Relaciones en los Hogares (Endireh) (Inegi, 2016). 


\section{Revista Iberoamericana \\ de las Ciencias Sociales y \\ Humanísticas}

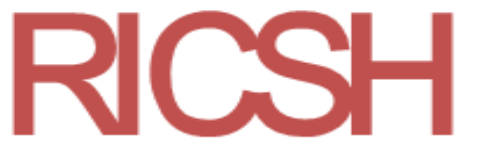

ISSN: $2395-7972$

El machismo y la misógina arraigadas en México de forma cultural vulneran todos los derechos de las mujeres al extremo de poner en peligro su integridad y provocar su muerte. La discriminación por género, impunidad, condición social, edad, etnia y criminalidad son aspectos también a considerar.

Esta investigación se fundamenta en la última reforma del 13 abril del 2018 a la Ley General de Acceso de las Mujeres a una Vida Libre de Violencia, la cual, en su artículo 1, a pie de letra dice:

Prevenir, sancionar y erradicar la violencia contra las mujeres, así como los principios y modalidades para garantizar su acceso a una vida libre de violencia que favorezca su desarrollo y bienestar conforme a los principios de igualdad y de no discriminación (H. Consejo de la Unión, 13 de abril del 2018, p. 1).

Además, en su artículo 5, inciso V, define a las modalidades de violencia como "las formas, manifestaciones o los ámbitos de ocurrencia en que se presenta la violencia contra las mujeres" (H. Consejo de la Unión, 13 de abril del 2018, p. 2). Ello para poner en conocimiento las diferentes manifestaciones y la frecuencia con que las viven las mujeres.

Respecto al interés de aplicar programas integrales para agresores, la directora del Instituto Nacional de las Mujeres (Inmujeres), Nadine Gassman, durante una presentación ante los medios en marzo del 2019, habló sobre la problemática y la eminente necesidad de erradicar el problema: por un lado, protegiendo a las mujeres del maltrato y, por otro, cambiando la forma de pensar del mexicano, incluyendo programas para trabajar la masculinidad (Valle, 2019). Hoy más que nunca es el momento de dar alternativas de tratamiento probadas científicamente y ejecutadas por varios años en otras sociedades semejantes a la mexicana y con aceptables índices de éxito, como es el Programa Galicia de Reeducación de Agresores de Género (Arce, Fariña, Vázquez, Novo y Seijo, 2015).

\section{Antecedentes del problema, violencia de género y el feminicidio en México}

Salvatierra (2007) menciona que el feminicidio es tan antiguo como el patriarcado. El feminicidio es ejecutado por hombres para asegurar el dominio masculino. Estas prácticas se han ido configurando debido a los arreglos sociales y políticos en culturas y en periodos diferentes.

El tema emergió contra criminales de la Segunda Guerra en Oriente, que sacó a la luz la masacre de civiles, en su mayoría mujeres vejadas y asesinadas con lujo de crueldad durante la ocupación japonesa en el sur de China en 1937. Aunque la querella feminista en torno a estos 


\section{Revista Iberoamericana \\ de las Ciencias Sociales y \\ Humanísticas}

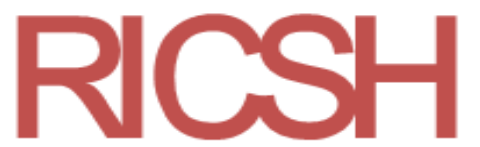

ISSN: 2395 - 7972

eventos incluía las violaciones masivas de niñas y jovencitas ocurridas en Bangladés (1971) y la matanza de jóvenes universitarias en Montreal. Tras este panorama, Diana Russell (citada en Romero, 2014) define los feminicidios como "los crímenes perpetrados contra las mujeres por el simple hecho de serlo" (párr. 22).

En cuanto a México, las evidencias y el registro sobre el feminicidio se han realizado de manera subsecuente desde el año 1993, cuando afloró este fenómeno en Ciudad Juárez, Chihuahua, como un problema recurrente. La información indica, igualmente, desplazamientos geográficos y rasgos regionales del fenómeno, que apuntarían hacia la existencia de contextos sociales, culturales, normativos, favorecedores de la violencia hacia las mujeres en sus modalidades más agresivas. La mayor indignación a la que están asociados estos eventos apunta a las autoridades responsables de perseguir y castigar estos delitos, cuya actuación acusa en la mayoría de los casos no solo omisión, sino incluso falta de sensibilidad y desprecio (Romero, 2014).

En el caso del Estado de México, los datos contenidos en las averiguaciones previas permiten afirmar que poco más de la mitad de las víctimas $(51 \%)$ se concentra en el rango de edad que va de los 16 a los 40 años, por lo que se trata del sector poblacional con mayor riesgo. Se trata, pues, de un amplio rango de edad que incluye a las mujeres en una de sus etapas más social y sexualmente activas. El resto de las víctimas (la otra mitad) se distribuye de la siguiente manera: $15 \%$ son mujeres de 0 a 15 años, y $34 \%$ tiene 40 años o más. Por lo que se refiere a su estado civil, la mayoría de las mujeres asesinadas son catalogadas, por declaraciones de familiares, amigos y testigos, dentro de algún tipo de relación de pareja, ya sea formal o informal (casada, divorciada, separada o en unión libre). En esta situación se encuentra $55 \%$ de las víctimas. Mientras que las mujeres reportadas simplemente como solteras abarcan $28 \%$ de las actas ministeriales revisadas. Del resto de las víctimas no se señala ningún tipo de dato al respecto. Cabe destacar que $58 \%$ de las mujeres que han sido objeto de violencia asesina tiene hijos, mientras que $27 \%$ no tiene descendencia. De aquellas mujeres que se sabe tenían hijos, $93 \%$ tuvo de uno a cuatro hijos. De este universo, $57 \%$ tuvo de uno a dos hijos, mientras que $33 \%$ tenía de tres a cuatro hijos, según lo reportado. El resto (10 \%) más de cuatro hijos (Salvatierra, 2007).

Existe varias fuentes que arrojan este tipo de cifras, por lo que no es del todo posible exponer datos precisos. Los datos recolectados van a ir de acuerdo con los objetivos de las distintas investigaciones realizadas, los cuales son derivados de las instituciones públicas en servicios sociales, de salud, cuerpos policiales, sistema de justicia, etc., que dan cuenta de los casos atendidos 


\section{Revista Iberoamericana \\ de las Ciencias Sociales y \\ Humanísticas}

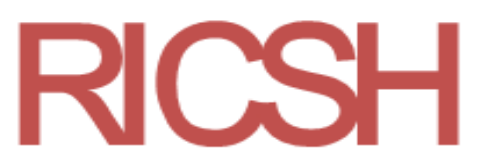

ISSN: 2395 - 7972

con propósitos fundamentalmente administrativos, pero que pueden ser empleados con fines estadísticos (Ramírez y Echarri, 2010).

Existe otra fuente de datos recabados sistemáticamente: el libro Ni una más. El feminicidio en México duele, de la periodista Frida Guerrera (2018), quien reporta el feminicidio de 1559 mujeres tan solo en 2016: mujeres "destazadas, calcinadas, violadas, asesinadas a balazos, abandonadas en: canales, ríos, terrenos, carreteras. Desaparecidas, despojadas, denigradas, olvidadas, descalificadas" (Guerrera, 2018, p. 21)

Asimismo, la periodista Guerrera (2018) comenta que es un fenómeno no tomado en su nivel de importancia por las autoridades y la sociedad. Y estima lo siguiente:

Solo el $18.79 \%$ de los feminicidios en el país fueron "investigados"; 1155 de estos no tienen ni responsables ni detenidos; 76 presuntos culpables se encuentran prófugos y 35 se suicidaron después de asesinar a sus parejas o exparejas sobre las mujeres asesinadas (Guerra, 2018 pp. 22).

Por otro lado, tenemos los registros de las instituciones gubernamentales. La Endireh (Inegi, 2003, 2006, 2011, 2016) es una fuente representativa sobre violencia contra las mujeres; recolecta datos de instituciones públicas y académicas, organismos internacionales y sociedad civil, donde se suman diferentes objetivos y perspectivas, lo que proporciona una visión panorámica del problema social y sus connotaciones. El objetivo fue encuestar a mujeres mayores de 15 años que fueran solteras "sin pareja" (viudas, divorciadas y separadas) y las casadas "con pareja" (unión a una o más parejas). Por lo que se incluye a todas las mujeres independientemente de su estado social reconocido.

La encuesta más reciente fue realizada con una muestra de 142363 viviendas, y se levantó del 3 de octubre al 18 de noviembre del 2016, con una tasa de respuesta de $85.7 \%$, y una cobertura geográfica nacional en áreas urbana y rural. La encuesta refiere que el grupo a estudiar son mujeres agredidas por su pareja por violencia emocional, física y sexual en los últimos 12 meses.

Ahí, la violencia de pareja se presentó en $43.9 \%$, uno de los porcentajes más altos, después de haber sufrido violencia a lo largo de la vida, que es de $66.1 \%$. Lo preocupante es que el principal agresor sea la pareja de la mujer por la cercanía y el dominio que se ejerce culturalmente. La agresión emocional está arriba con $40.1 \%$, le sigue la económica o patrimonial con $20.9 \%$, la agresión física con $17.9 \%$ y la sexual con $6.5 \%$. 


\section{Revista Iberoamericana \\ de las Ciencias Sociales y \\ Humanísticas}

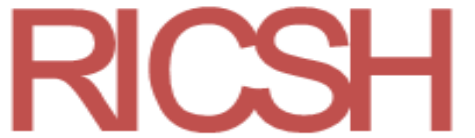

ISSN: $2395-7972$

\section{Impulso internacional para el tratamiento del feminicidio en México}

El comité de la Organización de las Naciones Unidas (ONU) ha instado al Gobierno de México a convertir en una cuestión prioritaria que se investigue y enjuicie adecuadamente a los perpetradores de los asesinatos contra mujeres. Entre las recomendaciones también se incluye la tipificación del feminicidio como delito en todos los estados mexicanos. Además, se aconseja la implementación de políticas para mitigar la trata de mujeres y niñas para la explotación sexual y el trabajo forzado. El organismo internacional indica que se deben implementar campañas para sensibilizar a las propias mujeres sobre sus derechos y la importancia de denunciar cualquier violencia de género.

De acuerdo con la Organización Mundial de la Salud (OMS) y la Organización Panamericana de la Salud (OPS):

¿Cuál es el mejor método para acabar con el feminicidio? Fortalecer la vigilancia y la detección del feminicidio y de la violencia por parte de la pareja.

Es preciso intensificar la recopilación y el análisis de datos sobre mortalidad, desglosar esos datos por sexo y, en el caso de los asesinatos, garantizar la documentación de la relación entre la víctima y el perpetrador. (...)

También se deben tomar medidas para elaborar y fortalecer métodos de investigación que mejoren el conocimiento del contexto social del feminicidio, incluidas las desigualdades por razón de género (OPS, 2013, p. 4).

Las instituciones y organizaciones internacionales se encuentran en alerta y sugieren a México diferentes alternativas ante el feminicidio, por ser el último paso de la agresión de la violencia de género. Como se ha visto, se trata de un evento que ocurre a diario y frecuentemente queda impune por el poco control que existe, lo cual produce un círculo vicioso: por un lado, son pocas las denuncias de violencia de género y, por el otro, cuando las hay, pocos castigos. Lo anterior provoca que la población se muestre indiferente ante la situación o lo encuentre como algo cotidiano.

Precisamente, sabiendo que el feminicidio es un evento con alta frecuencia en México, el Comité para la Eliminación de la Discriminación contra la Mujer (Cedaw) de las ONU ha pedido que esta nación "adopte medidas urgentes" para prevenir las muertes violentas, los homicidios y las desapariciones forzadas de mujeres (Martínez, 3 de agosto de 2018). De hecho, México se encuentra entre los primeros cinco países de Latinoamérica con más feminicidios. La coordinadora ejecutiva del Observatorio Ciudadano Nacional del Feminicidio, que colabora con ONU Mujeres, 


\section{Revista Iberoamericana \\ de las Ciencias Sociales y \\ Humanísticas}

ISSN: $2395-7972$

María de la Luz Estrada, comenta al respecto: "La violencia que vivimos de discriminación cotidiana la tienes ahí o la violencia doméstica que la autoridad no pudo prevenir, en eso puede haber un mayor control” (Martínez, 3 de agosto de 2018, párr. 7).

\section{La vulnerabilidad aceptada e íntima}

Existen diferentes tipos de feminicidio — debido a que, si bien no cambia la premisa general, a saber, por razones de género, las circunstancias en las que suceden estos delitos cambian de modus operandi- . Así, tomando en cuenta la investigación de Julia Monárrez (2009, citada en Olamendi, 2016) en Ciudad Juárez, y otros estudios sobre el fenómeno de violencia en contra de las mujeres, específicamente su forma más extrema como la violencia feminicidio, se han catalogado 11 modalidades. Y de entre ellas este estudio ha seleccionado una: La vulnerabilidad aceptada íntima.

Es la muerte de una mujer cometida por un hombre con quien la víctima tenía o había tenido una relación o vinculo intimo: marido, exmarido, compañero, novio, exnovio o amante. Esta relación en algún momento fue aceptada o se dio permiso para estar en relación (Olamendi, 2010, p. 35).

En este sentido, los crímenes de feminicidios son, claramente, crímenes de poder, es decir, crímenes cuya dupla función es, en este modelo, simultáneamente, la retención o manutención y la reproducción del poder (Segato, 2006).

El feminicidio no se entiende como un hecho aislado y cerrado en sí mismo. Son construcciones sociales tradicionales a través de las cuales hombres y mujeres se relacionan y funciona como un detonador que explicaría en gran parte el incremento de la violencia contra las mujeres. De esta forma, los feminicidios, y la violencia que los acompaña, no son solo la expresión de una crisis (social, económica o de valores), sino una respuesta al proceso de construcción de las mujeres como sujetos (Rodríguez, 2011).

El feminicidio es un fenómeno inserto en un escenario social particular, al que se le suma la alteración de los valores y normas masculinos por el trabajo que realizan las mujeres sobre su subjetividad, lo cual les permite convertirse en sujetos de deseo, con relaciones, y conciencia, así como en motores de integración del "yo" y el mundo.

Es el resultado de una masculinidad trastocada por la constante consolidación del trabajo de las mujeres sobre sí mismas, persiguiendo ser sujeto para dejar de ser objeto. 


\section{Revista Iberoamericana \\ de las Ciencias Sociales y \\ Humanísticas}

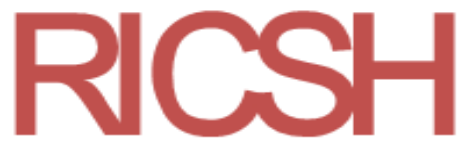

ISSN: $2395-7972$

La violencia asesina es una forma en que el victimario niega la subjetividad de su víctima, cosificándola para reafirmar la suya propia. De alguna forma, en el caso de los feminicidios, esto muestra que en la acción donde un hombre mata a una mujer se niega algo de ella y se afirma algo de él (Moscovici, 2002).

\section{El perfil del victimario/agresor primario de la pareja o cónyuge}

Como se puede ver, existen muchos estudios sobre las víctimas y al victimario se le considera como el oportunista que abusa de las condiciones para ejercer ese dominio.

El victimario (agresor primario) es considerado como un delincuente que ejerce su dominio sobre su pareja sin causa aparente y ha sido tipificado muchas veces como enfermo mental que no controla sus impulsos agresivos en contra de la figura femenina.

Se sabe que el instinto agresivo tiene un carácter de supervivencia no solo en animales, sino también en el ser humano, pero este último requiere manejarlo para su adaptación social. Entonces, cuando no se maneja la agresión, se considera una patología, y está estipulado en el DSM-5 dentro de los trastornos de relación humana. Consecuentemente, los comportamientos violentos en los animales no se consideran negativos, pero sí son negativos en los seres humanos, aunque será para conservar su identidad, estatus social, nivel económico o su vida. Por ser formas de expresión no aceptada por la sociedad y es censurable y la mayoría de las veces condenable (APA, 2013).

La violencia se asocia a aspectos físicos (deformes) o a mentales (psicópatas) no aceptables a la sociedad. La dinámica frecuentemente expresada en las parejas surge a raíz de una expresión de agresión unida a una expresión de arrepentimiento y de enmienda de los daños producidos. Lo que el violentador pretende con ello es conciliarse con la víctima y con la sociedad. Entonces, el victimario presenta sentimientos de culpa y autocrítica, que lo hacen actuar de tal manera. En otras veces emplea la autojustificación, la racionalización para justificar su agresividad en contra de la víctima. Porque ellos están conscientes que están mal su conducta agresiva y socialmente es castigada (Espada y Torres, 1996).

En diversos estudios se ha comprobado que la conducta violenta de los agresores primarios es frecuentemente aprendida porque han vivido en carne propia las acciones de otros agresores o que han visto la relación agresiva en parejas, todas bajo el manto de la impunidad. Se trata de un doble mensaje: la conducta agresiva en el contexto privado (casa, escuela, hasta trabajo) a veces es censurada pero no es castigada, y en otras ocasiones incluso es premiada, porque se conserva el estatus de poder del victimario (Espada y Torres, 1996; López, 2004). 


\section{Revista Iberoamericana \\ de las Ciencias Sociales y \\ Humanísticas}

ISSN: $2395-7972$

Dentro de la vida íntima o del seno familiar se crea un sistema de poder y de jerarquía que es respetado por la dinámica de los integrantes debido a creencias y estilos de vida que hacen que la familia opere (Espada y Torres, 1996; López, 2004).

Las dinámicas familiares agresivas en contra de la mujer se generan por la desigualdad (menor fuerza física, dependencia económica, menor nivel académico, menos relaciones sociales, menor apoyo familiar, etc.), lo que hace que la mujer conserve su rol de subordinada dentro de la familia, manifestando conductas de tolerancia, miedo y de sacrificio ante el mal trato para que continúe operando la familia. Estas conductas permisivas hacen que el agresor primario siga con su conducta agresiva y lo considere parte de su relación con su pareja, y cuando el control se sale de su dominio ejerce más control para no perder la dinámica preestablecida. Aquí es cuando la agresión empieza a escalar y puede hasta llegar al feminicidio (López, 2010).

\section{Tipificación legal del feminicidio en México}

En México, el artículo 325 del Código Penal Federal mexicano (Diario Oficial de la Federación [DOF], 12 de abril de 2019) dispone lo siguiente:

Comete delito de feminicidio quien prive de la vida a una mujer por razones de género. Se considera que existen razones de género cuando concurra alguna de las siguientes circunstancias:

I. La víctima presente signos de violencia sexual de cualquier tipo.

II. A la víctima se le hayan infligido lesiones o mutilaciones infamantes o degradantes, previas o posteriores a la privación de la vida o actos de necrofilia.

III. Existan antecedentes o datos de cualquier tipo de violencia en el ámbito familiar, laboral o escolar, del sujeto activo en contra de la víctima.

IV. Haya existido entre el activo y la víctima una relación sentimental, afectiva o de confianza.

V. Existan datos que establezcan que hubo amenazas relacionadas con el hecho delictuoso, acoso o lesiones del sujeto activo en contra de la víctima.

VI. La víctima haya sido incomunicada, cualquiera que sea el tiempo previo a la privación de la vida.

VII. El cuerpo de la víctima sea expuesto o exhibido en un lugar público.

A quien cometa el delito de feminicidio se le impondrán de cuarenta a sesenta años de prisión y de quinientos a mil días multa. Además de las sanciones 


\section{Revista Iberoamericana \\ de las Ciencias Sociales y \\ Humanísticas}

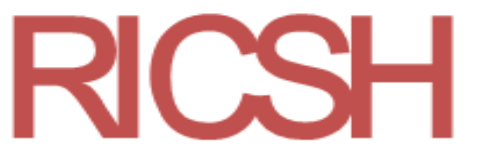

ISSN: $2395-7972$

descritas en el presente artículo, el sujeto activo perderá todos los derechos con relación a la víctima, incluidos los de carácter sucesorio.

En caso de que no se acredite el feminicidio, se aplicarán las reglas del homicidio.

Al servidor público que retarde o entorpezca maliciosamente o por negligencia la procuración o administración de justicia se le impondrá pena de prisión de tres a ocho años y de quinientos a mil quinientos días multa, además será destituido e inhabilitado de tres a diez años para desempeñar otro empleo, cargo o comisión públicos.

Según el Observatorio Ciudadano Nacional del Feminicidio [OCNF] (2014), el análisis de su problemática y su conceptualización se originan a partir de una perspectiva sociológicaantropológica; en México se avanzó hacia una perspectiva jurídica que permitió llegar a su tipificación en el ámbito penal. Los expertos señalan que el feminicidio deriva del odio relacionado con el género de la víctima, el cual se evidencia con la mutilación de los cuerpos, con signos de violación sexual o tortura en ellos y con el hecho de cómo intentan esconderlos en terrenos o canales.

Aunque en México se han realizado grandes avances con respecto a la adopción de estándares internacionales y leyes específicas de protección a los derechos de las mujeres en la legislación interna, sigue siendo un tema pendiente dado los altos índices de violencia en contra de ellas.

Hay acciones en el marco legal que realiza el Inmujeres. Por ejemplo, en octubre del 2018 publica la creación del programa de "Alerta de violencia de género contra las mujeres", un mecanismo de protección de los derechos humanos apoyado en la Ley General de Acceso de las Mujeres a una Vida Libre de Violencia generada en 2009 y su última actualización realizada en 2018. la cual contiene un conjunto de acciones gubernamentales de emergencia para enfrentar y erradicar la violencia feminicida o la existencia de un agravio comparado que impida el ejercicio pleno de los derechos humanos de las mujeres.

El Inegi, a través de la Endireh, realizada desde 2003 al 2016, se percata que la mayor incidencia de agresión de género se encuentra en los hogares. Y para disminuir el problema crea la Guía de autocuidado para tu seguridad emocional (Endireh, 2016), que implica un conjunto de acciones intencionadas para controlar o mitigar los factores internos o externos que pueden, eventualmente, comprometer su salud y seguridad. 


\section{Revista Iberoamericana \\ de las Ciencias Sociales y \\ Humanísticas}

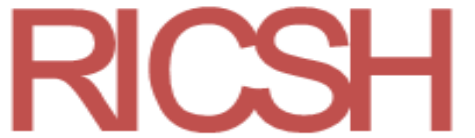

ISSN: $2395-7972$

\section{Implicaciones psicológicas y sociales sobre fenómeno del feminicidio}

Russell y Radford (2006) consideran la misoginia, el sentido de superioridad de género y la concepción de las mujeres como posesión como las variables que se transmiten culturalmente y que favorecen la violencia de los hombres hacia las estas.

Según Arce y Fariña (2010), los agresores de género se definen por determinadas características sociolegales específicas, a saber:

- $\quad$ Son primarios.

- Han cometido delitos contra las personas en el ámbito privado.

- $\quad$ Las agresiones se derivan del ejercicio del poder asociado al género masculino.

- La violencia ejercitada puede ser de muy diversos tipos (física, psíquica, sexual, amenazas, coacciones, privación de la libertad).

- La violencia no tiene por qué estar presente en otros ámbitos.

- $\quad$ Se caracterizan por una alta probabilidad de reagresiones (violencia continuada) y de recaídas.

- Se espera un cierto índice de patología dual, esto es, la combinación de violencia de género con otra patologías como alcoholismo, drogodependencia, ludopatía, daños en las funciones neropsicológicas o psicopatología.

Bejarano (2014), en su artículo del "El feminicidio es solo la punta del iceberg”, menciona que al realizar dicho estudio combinó métodos cualitativos y cuantitativos, y retomó algunas entrevistas a víctimas, así como resultados de grupos focales con personas expertas, para estudiar las particularidades de la situación de violencia contra las mujeres en la zona noroeste de México, la problemática general de la violencia feminicida, con énfasis en dos aspectos: 1) las manifestaciones de la violencia de múltiples y continuas formas en la vida de las mujeres y 2) los avances en la tipificación del feminicidio como delito autónomo y los obstáculos en el acceso a la justicia para las víctimas, como parte de la violencia institucional que permite la existencia del fenómeno. En este estudio se concluye que el feminicidio es solo la punta del iceberg, como se anticipa en su título: la violencia hacia las mujeres debe ser atendida de manera integral, pues bajo él se cimienta una argamasa de violencias que les impiden su ejercicio pleno de derechos.

Castañeda, Ravelo y Pérez (2013), por su parte, exponen la constitución de las organizaciones de madres de mujeres asesinadas y desaparecidas en la lucha por la justicia en Ciudad Juárez y de la Audiencia de Feminicidio y Violencia de Género, que se ha conformado en 


\section{Revista Iberoamericana \\ de las Ciencias Sociales y \\ Humanísticas}

ISSN: $2395-7972$

el marco del Tribunal Permanente de los Pueblos, haciendo énfasis en la importancia de atender la violencia de género contra las mujeres en todas sus expresiones, pues no se trata de "un grupo social más", sino de la mitad de la población nacional que, por lo tanto, es a la vez un grupo específico que recibe formas particulares de maltrato y crueldad.

La impunidad que marca este caso responde a un contexto social que tolera y reproduce la violencia contra las mujeres, manteniendo formas de discriminación basadas no solo en el género, sino también en el origen étnico y de clase, la ausencia de un compromiso real por parte del Estado mexicano durante 17 años, para investigar, sancionar y ofrecer una efectiva reparación del daño permite la creación de un contexto de impunidad (Macleod et al., 2012, citado en Castañeda et al., 2013, p. 30).

\section{Preguntas de investigación}

- ¿ ¿La narrativa de los agresores primarios mostrará los disparadores que ayudarán a conducir de manera certera pláticas o tratamientos psicoterapéuticos?

- ¿Es posible que las pláticas o los tratamientos contengan los elementos que modifiquen conceptos y conductas para disminuir la agresión hacia el género femenino?

\section{Material}

Los métodos científicos para evaluar al agresor primario de la violencia de género en México no son muy usuales. Existen entrevistas estructuradas y semiestructuradas para evaluar a este tipo de relación con poblaciones europeas o estadounidenses, las cuales muestran niveles de validez y confiabilidad.

Las evaluaciones normalmente se realizan de forma individual para cada uno de los integrantes de la pareja, y se obtiene un diagnóstico personal de problemas de relaciones interpersonales hasta llegar al conflicto con la pareja, porque esto se considera como un resultado de su desadaptación social.

Uno de los instrumentos que evalúa el conflicto de pareja crónico es el Inventario de satisfacción marital (MSI-R, por sus siglas en inglés), elaborado por Whisman, Snyder y Beach (2009), donde se describe el nivel de cronicidad del padecimiento y las áreas donde se encuentra 


\section{Revista Iberoamericana \\ de las Ciencias Sociales y \\ Humanísticas}

ISSN: 2395 - 7972

un mayor conflicto para ser tratado en terapia de pareja. El instrumento se encuentra adaptado y estandarizado para población de habla hispana, inglesa y alemana.

Otro instrumento es el MSI-B, que puede ser autoaplicable y está disponible en Western Psychological Services. Fue elaborado con una muestra de Estados Unidos y dos muestras alemanas (Klatt, Hahlweg, Job y Foran, manuscrito no publicado). Su aplicación y diagnosis es sencilla; detecta cómo se encuentra la relación entre la pareja y el nivel de patología de cada uno de sus miembros.

Ambos instrumentos refieren que sean complementados con una diagnosis integral porque el conflicto de pareja se considera como un síntoma de la patología conductual social.

Por lo sutil de la investigación y lo complejo del tema a estudiar, se decidió tener un análisis mixto (cuantitativo y cualitativo) para triangular la información y tener una visión completa del fenómeno. Así, poder obtener un diagnóstico y una visión del participante (agresor primario de la violencia de género) ante su relación de pareja.

1) La primera entrevista fue estructurada, a través del $D S M-5$, utilizando el diagnóstico "Relación conflictiva con el cónyuge o pareja".

Los manuales de diagnóstico como en el DSM-5 contienen el código V, el cual se refiere a los problemas de tipo psicosocial que están influyendo sobre la salud mental del paciente. Entre estos problemas se ubica la descripción conflictiva con el cónyuge o pareja, que evalúa los siguientes factores: 1) problemas conductuales (dificultades en la resolución de conflictos, abandono, distanciamiento emocional e intromisión excesiva), 2) problemas cognitivos (atribuciones negativas constantes en relación a las intenciones de la pareja y rechazo constante a las conductas positivas de la pareja) y 3) tristeza, apatía o rabia crónicas hacia la pareja. Ambas versiones de evaluación miden existencia o no existencia de estos por un periodo de tres a seis meses, considerando leve, de 6 a 12 meses, que es considerado como moderado, y más de 12 meses, cuando ya es calificado como grave (APA, 2013).

2) La segunda fue una entrevista semiestructurada para maltratadores de género, por ser una herramienta diagnóstica que evalúa el área psicosocial. Esta entrevista implica: 1) valoración del control emocional (existencia o no existencia); 2) autoconcepto (bueno, regular y malo) y estrategias de afrontamiento (escape o enfrentar); 3) ideas o concepto hacia la pareja (buenas, regular o malas), y 4) comportamientos desadaptados por adiciones (eventuales o frecuentes). También refiere la descripción de los episodios de maltrato medio a través: 1) frecuencia (veces por semana, 2) duración (minutos u horas), 3) intensidad (leve, moderado y fuerte), 4) 


\section{Revista Iberoamericana \\ de las Ciencias Sociales y \\ Humanísticas}

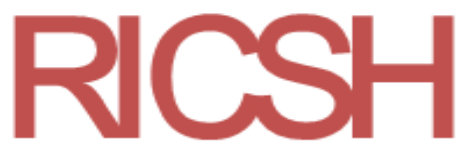

ISSN: $2395-7972$

consecuencias de los episodios violentos (leve, regular y grave) y 5) asunción de responsabilidad (existencia o no existencia) (Arce y Fariña, manuscrito no publicado).

\section{Método}

Se trata de un estudio no experimental, con diseño ex post facto, retrospectivo, con análisis mixto cuantitativo descriptivo y cualitativo fenomenológico y con enfoque etnometodológico, para una muestra no probabilística constituida por 32 participantes, evaluados por los servicios psicológicos con diagnóstico de "Relación conflictiva con el cónyuge o pareja" (criterios del DSM5), referidos por los Centros de Atención de la Casa de la Mujer de los municipios de Atizapán y Ecatepec, Estado de México, México.

El análisis estadístico descriptivo (informa el qué y su frecuencia) fue aplicado en los siguientes puntos: 1) las características sociodemográficas de la muestra; 2) los criterios de diagnósticos señalados en el DSM-5 (APA, 2013), en el apartado "Relación conflictiva con el cónyuge o la pareja": entrevista estructurada con respuesta dicotómica; 3) las características del área psicosocial y episodios de maltrato estipuladas en la entrevista semiestructurada para maltratadores de género, con respuesta de opción múltiple (Arce y Fariña, manuscrito no publicado).

El análisis cualitativo de tipo fenomenológico y enfoque etnometodológico fue aplicado a la explicación (información del cómo) que daban los participantes a sus respuestas, incluidas en la entrevista semiestructurada para maltratadores de género (Arce y Fariña, manuscrito no publicado).

El objetivo de este estudio se encuentra enfocado a los fenómenos sociales desde la perspectiva de los actores, utilizando dos entrevistas diagnósticas, a saber, una estructurada y otra semiestructurada, para entender el qué y el cómo de la realidad social, poniendo como eje la cotidianeidad del sujeto, circunstancias que forman al fenómeno (Urbano, 2007).

La población y la muestra fue la misma porque se entrevistaron a 32 participante por ser emitidos por los Centros de Atención y Reeducación para Personas que Ejercen Violencia de Género, provenientes de los municipios de Atizapán y Ecatepec, como ya se mencionó líneas atrás. Los participantes ya tenían un expediente, donde se solicitaba pláticas o tratamiento psicológico, emitido al Consejo Estatal de la Mujer y Bienestar Social. 


\section{Revista Iberoamericana \\ de las Ciencias Sociales y \\ Humanísticas}

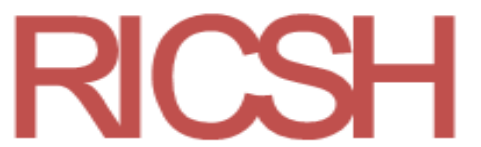

ISSN: $2395-7972$

\section{Procedimiento}

1) Se realizó una investigación documental de los expedientes de los participantes para indagar de los antecedentes generales de la violencia de género y del perfil del agresor primario para entender la dinámica del conflicto intrafamiliar y de los participantes.

2) Se proporcionó a los participantes la hoja de consentimiento informado y responsabilidad para ser firmada. La entrevista se realizó bajo los lineamientos del Código ético del psicólogo (Sociedad Mexicana de Psicología, 2010).

3) Se realizó el estudio etnológico a través de dos entrevistas. La primera una entrevista estructurada apoyado en la guía del DSM-5 (APA, 2013), utilizando el diagnóstico de "Relación conflictiva con el cónyuge o pareja. Y la segunda una entrevista semiestructurada para maltratadores de género, que mide la valoración del control emocional, el autoconcepto, las ideas o conceptos hacia la pareja, los comportamientos desadaptados y solicita una descripción de los episodios de maltrato (Arce y Fariña, manuscrito no publicado).

4) Se levantaron datos por caso y respetando la estructura y secuencia de cada una de las entrevistas. La segunda entrevista permitió obtener diálogos a través de ejes temáticos de interés, los cuales fueron influenciados por las experiencias particulares de los participantes. Así, se le dio a los datos un enfoque etnometodológico, donde cada participante podía expresar su dinámica familiar y su vida cotidiana.

5) Se analizaron los datos con estadística descriptiva de las características de la muestra y los ítems de ambas entrevistas.

6) Se realizó un análisis fenomenológico de las grabaciones previamente cargadas en el programa Atlas.ti, en su versión 7.5.4; se situaron como ejes las áreas ya evaluadas para dar una guía de jerarquización de los temas de acuerdo con las vivencias de cada uno de los participantes.

\section{Resultados}

Las características sociodemográficas con mayor frecuencia en la muestra son las siguientes: participantes del género masculino entre 26 a 35 años, nivel académico básico (50 \%), estado civil solteros (69 \%) y nivel socioeconómico medio (47 \%) (ver tabla 1). 


\section{Revista Iberoamericana \\ de las Ciencias Sociales y \\ Humanísticas}

Tabla 1. Características sociodemográficas de la muestra

\begin{tabular}{|c|c|c|c|c|c|}
\hline \multicolumn{6}{|l|}{$n=32$} \\
\hline \multirow[b]{2}{*}{ Rango } & \multicolumn{2}{|l|}{ Edad } & \multicolumn{3}{|c|}{ Nivel académico } \\
\hline & Total & $\%$ & Básico & Medio & Superior \\
\hline 15-25 años & 7 & $22 \%$ & 3 & 2 & 2 \\
\hline 26-35 años & 11 & $34 \%$ & 6 & 4 & 3 \\
\hline 36-45 años & 10 & $31 \%$ & 5 & 5 & 0 \\
\hline 46-55 años & 4 & $13 \%$ & 2 & 2 & 0 \\
\hline 56-más años & 0 & $0 \%$ & 0 & 0 & 0 \\
\hline Totales & 32 & $100 \%$ & 16 & 13 & 5 \\
\hline \multicolumn{3}{|l|}{ Porcentajes } & $50 \%$ & $41 \%$ & $16 \%$ \\
\hline & \multicolumn{2}{|c|}{ Estado civil } & \multicolumn{3}{|c|}{ Nivel socioeconómico } \\
\hline Rango & Casados & Solteros & Bajo & Medio & Alto \\
\hline 15-25 años & 2 & 3 & 2 & 3 & 1 \\
\hline 26-35 años & 4 & 3 & 4 & 4 & 0 \\
\hline 36-45 años & 3 & 9 & 4 & 7 & 2 \\
\hline 46-55 años & 1 & 7 & 3 & 1 & 1 \\
\hline 56-más años & 0 & 0 & 0 & 0 & 0 \\
\hline Totales & 10 & 22 & 13 & 15 & 4 \\
\hline Porcentajes & $31 \%$ & $69 \%$ & $41 \%$ & $47 \%$ & $13 \%$ \\
\hline
\end{tabular}

Fuente: Elaboración propia

\section{Análisis cuantitativo}

Las entrevistas estructuradas basadas en el DSM-5 (APA, 2013) muestran mayor frecuencia en los criterios puestos a continuación: 1) Rechazo constante a las conductas positivas de la pareja (84 \%) y 2) Dificultades en la resolución de conflictos (72\%), acompañadas por una tristeza, apatía y/o rabia crónicas hacia la pareja, lo que provoca el abandono, distanciamiento emocional con la pareja (ambas con $66 \%$ ) (ver tabla 2). 
Revista Iberoamericana

de las Ciencias Sociales y

Humanísticas

ISSN: $2395-7972$

Tabla 2. DSM-5, Relación Conflictiva con el Cónyuge o Pareja

\begin{tabular}{|l|l|l|l|l|}
\hline \multicolumn{1}{|c|}{$n=32$} & Sí & $\%$ & No & $\%$ \\
\hline \multicolumn{1}{|c|}{ Priterios de diagnóstico } & & & & \\
\hline \multicolumn{1}{|c|}{ Problemas conductuales } & 23 & $72 \%$ & 9 & $28 \%$ \\
\hline $\begin{array}{l}\text { Dificultades en la resolución de conflictos } \\
\text { Abandono, distanciamiento emocional }\end{array}$ & 21 & $66 \%$ & 11 & $34 \%$ \\
\hline $\begin{array}{l}\text { Intromisión excesiva } \\
\text { Progativas constantes en relación con las }\end{array}$ & 12 & $38 \%$ & 20 & $63 \%$ \\
\hline $\begin{array}{l}\text { Atribuciones } \\
\text { intenciones de la pareja }\end{array}$ & & $56 \%$ & 14 & $44 \%$ \\
\hline \begin{tabular}{l} 
Rechazo constante a las conductas positivas de la pareja \\
\hline Pristeza, apatía y/o rabia crónicas hacia la pareja
\end{tabular} & 27 & $84 \%$ & 5 & $16 \%$ \\
\hline
\end{tabular}

Fuente: Elaboración propia

La entrevista semiestructurada para maltratadores de género ayuda a entender los fenómenos sociales desde la perspectiva de los actores, el cómo de la realidad social, poniendo como eje la cotidianeidad del sujeto, circunstancias que forman al fenómeno (Urbano, 2007). Como resultado de este apartado se observan frecuentes afrontamientos (75\%) por su bajo control de impulsos $(56 \%)$ y malas ideas sobre la mujer y comportamientos desadaptados por adicciones (ambos rubros con $53 \%$ ) (ver tabla 3 ). 


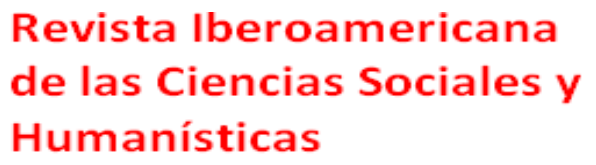

ISSN: 2395 - 7972

Tabla 3. Entrevista semiestructurada para maltratadores de género

\begin{tabular}{|c|c|c|c|c|c|c|}
\hline \multicolumn{7}{|c|}{$n=32$} \\
\hline $\begin{array}{l}\text { Área } \\
\text { Psicosocial }\end{array}$ & Existencia & $\%$ & No existencia & $\%$ & & $\%$ \\
\hline \multirow[t]{2}{*}{$\begin{array}{l}\text { Control } \\
\text { emocional }\end{array}$} & 14 & $\begin{array}{l}44 \\
\%\end{array}$ & 18 & $56 \%$ & & \\
\hline & Bueno & & Regular & & Malo & \\
\hline \multirow[t]{2}{*}{ Autoconcepto } & 3 & $9 \%$ & 19 & $59 \%$ & 10 & $31 \%$ \\
\hline & Escapa & & Enfrenta & & & \\
\hline \multirow[t]{2}{*}{ Afrontamiento } & 8 & $\begin{array}{l}25 \\
\%\end{array}$ & 24 & $75 \%$ & & \\
\hline & Buenas & & Regular & & Malas & \\
\hline \multirow[t]{2}{*}{$\begin{array}{l}\text { Ideas o } \\
\text { concepto hacia } \\
\text { la pareja }\end{array}$} & 6 & $\begin{array}{l}19 \\
\%\end{array}$ & 9 & $28 \%$ & 17 & $53 \%$ \\
\hline & Frecuentes & & Eventuales & & & \\
\hline $\begin{array}{l}\text { Comportamien } \\
\text { tos } \\
\text { desadaptados } \\
\text { por adiciones }\end{array}$ & 15 & $\begin{array}{l}47 \\
\%\end{array}$ & 17 & $53 \%$ & & \\
\hline
\end{tabular}

Fuente: Elaboración propia

La entrevista semiestructurada para maltratadores de género también ayuda a entender los fenómenos sociales del maltrato a la víctima desde la descripción de los actores, el cómo se ejerce el maltrato (Urbano, 2007). Aquí se observa la no asunción de responsabilidad (66\%), con un rango de tres a cuatro episodios violentos por semana (44\%), con una duración de minutos ( $88 \%$ ), intensidad leve $(75 \%)$ y con consecuencias leves (63\%) (ver tabla 4). 


\section{Revista Iberoamericana \\ de las Ciencias Sociales y \\ Humanísticas}

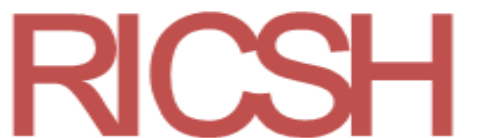

ISSN: 2395 - 7972

Tabla 4. Descripción de los episodios de maltrato

\begin{tabular}{|c|c|c|c|c|c|c|}
\hline \multicolumn{7}{|c|}{$n=32$} \\
\hline Episodios de maltrato & $0-2$ & & 3 a 4 & & 5 a 7 & \\
\hline \multirow{2}{*}{$\begin{array}{l}\text { Frecuencia de episodios } \\
\text { violentos en una semana }\end{array}$} & 5 & $16 \%$ & 14 & $44 \%$ & 13 & $41 \%$ \\
\hline & Minutos & & Horas & & Días & \\
\hline \multirow[t]{2}{*}{ Duración por episodio } & 28 & $88 \%$ & 4 & $13 \%$ & 0 & \\
\hline & Leve & & Moderado & & Fuerte & \\
\hline \multirow[t]{2}{*}{ Intensidad } & 24 & $75 \%$ & 5 & $16 \%$ & 3 & $9 \%$ \\
\hline & Leve & & Regular & & Grave & \\
\hline \multirow{2}{*}{$\begin{array}{c}\text { Consecuencias de los episodios } \\
\text { violentos }\end{array}$} & 20 & $63 \%$ & 10 & $31 \%$ & 2 & $6 \%$ \\
\hline & Sí & & No & & & \\
\hline Asunción de responsabilidad & 11 & $34 \%$ & 21 & $66 \%$ & & \\
\hline
\end{tabular}

Fuente: Elaboración propia

\section{Análisis cualitativo, fenomenológico}

Se realizaron grabaciones para conocer de viva voz el cómo de las respuestas realizadas en la entrevista semiestructurada.

A continuación se enlistan algunos aspectos sobresalientes obtenidos a partir de dichas grabaciones:

1) Ante la acción se observa que los agresores de género presentan frecuentes conductas de afrontamiento y comentan que ellos son provocados por la situación o por la pareja, que también los agrede y muchas veces son respuestas a la previa agresión recibida, y que su pareja o familiares de estas lo maltratan. Respecto a las adicciones, comentan que si las personas que lo rodean saben que ellos no se encuentran en condiciones físicas para reaccionar correctamente que no los molesten.

2) Ante la no asunción de responsabilidad agresores de género tenían respuestas evasivas; justificaban sus acciones con eventos causantes del conflicto o descargaban la culpa en su pareja. 


\section{Revista Iberoamericana \\ de las Ciencias Sociales y \\ Humanísticas}

ISSN: $2395-7972$

3) Ante la frecuencia e intensidad de los episodios violentos, los agresores de género comentaban que existían problemas continuos con la pareja y que muchas veces no respondían a la agresión externa, pero que la situación o la pareja lo incitaban a realizar los impulsos verbales o físicos inadecuados. Los conflictos siempre duraban minutos y que su respuesta estaba en la proporción del momento.

\section{Discusión}

La investigación mixta cuantitativa y cualitativa nos ayudó a conocer de una forma triangulada el fenómeno social de la violencia de género desde la perspectiva de los actores (agresores primarios de violencia de género) y entender cómo se desarrolla la violencia en la relación de pareja desde el relato del victimario/agresor primario. La postura del este es de víctima de la sociedad y de las circunstancias, que lo inducen a que cometa actos de violencia.

El victimario/agresor primario siempre tuvo excusas ante sus actos violentos. Como estudiosos de la salud mental podemos señalar que la "no responsabilidad ante actos violentos sociales" está íntimamente ligada a conflictos internos de devaluación de su integridad y disminución de valores ante su propia persona y de las personas que lo rodean (Espada y Torres, 1996; Lila, Gracia y Herrero, 2012).

La cultura machista y misógina lo disculpa: el agresor de género está cumpliendo con sus derechos de control, "cuidado" de la pareja, porque culturalmente ha aprendido que la mujer no tiene las capacidades físicas ni mentales para valerse por sí misma, considerándola "débil y desprotegida", pensando que el mundo siempre la rebasa. Para evitar conflictos futuros con el mundo externo es mejor prevenir controlando su mundo íntimo o privado (Arce, Fariña, y Novo 2014).

El "cuidado" y control muchas veces es para que no sea apropiada por otros seres del género masculino, que viven al acecho de su pareja, lo que provoca escenas de celos, para que no se vulnere lo que el agresor considera propio (Lila et al., 2012).

La cuestión de los valores lo presentan desequilibrado, porque el "valor de tener" es mayor que el "valor de ser", por lo que su identidad enfrenta una crisis. Su valor de ser "hombre" depende de lo que posee y la mujer es considerada un objeto y entre más demuestre a la sociedad el dominio de sus posesiones va a valer más (Jelev, 2006). Esto también se ve relacionado con la bigamia: entre más mujeres tenga y domine "más vale". 


\section{Revista Iberoamericana \\ de las Ciencias Sociales y \\ Humanísticas}

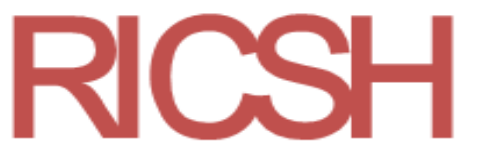

ISSN: $2395-7972$

Los participantes se quejan frecuentemente de la liberación femenina y la diversidad de género. Ambos fenómenos son los principales disparadores de la agresión.

La liberación femenina provoca en la mujeres rebeldía y pérdida de roles (madre, esposa, compañera y pareja sexual). Las mujeres no ejecutan estos como dicen la tradición y las costumbres, lo que hace que la familia pierda fuerza, orden y nivel jerárquico (Espada y Torres, 1996).

La diversidad de género es considerado como una aberración del ser humano, que no debe de ser permitida, porque es una falta de respeto a la masculinidad y conduce al libertinaje.

La falta de adaptación social genera inconformidad, que es confrontada por agresividad o evadida a través del alcohol o drogas. Esta última genera adiciones que vuelve a confrontar con la familia y la sociedad (Espada y Torres, 1996).

Sus ideas arraigadas son producto de sus vivencias en la infancia y culturalmente aprendidas, por lo que requieren de pláticas o psicoterapia para que se den cuenta que lo que ellos consideran como cierto y difícil de cambiar es posible, y que trae beneficio para él, su pareja y la familia (Castillo, 2004).

A partir de las entrevistas realizadas como parte de este estudio se observan sujetos con talante ansioso-depresivo. Sujetos que sufren, miedosos a perder control y tristes por no ser empáticos con su pareja o con personas que los rodean. El negativismo les impulsa a mostrarse agresivos, conceptos considerados como patológicos y estipulados en el DSM-5, dentro de los trastornos de relación humana (APA, 2013).

Se debe concretar la oportunidad de ofrecerle a los maltratadores de género los programas de justicia terapéutica para agresores de género, que tratan el núcleo de la violencia de pareja. En este estudio se ha demostrado que los agresores no son enfermos mentales que actúan sin responsabilidad del acto, sino sujetos que tienen problemas conductuales y relaciones humanas, generados por pensamientos aprendidos de minusvalía para el género femenino, lo que evita la equidad de género (Arce et al., 2015; Pérez, Giménez y Espinoza, 2012).

Las limitaciones del estudio fueron la asistencia forzada por sentencia jurídica, lo que propiciaba justificar sus actos. En los espacios físicos asignados, además, a veces no se tenían la privacidad requerida. 


\section{Revista Iberoamericana \\ de las Ciencias Sociales y \\ Humanísticas}

ISSN: $2395-7972$

La fortaleza está en analizar de primera mano lo que piensa el agresor y que lo impulsa a cometer el delito. Esta información va ayudar a dar un tratamiento certero e individualizado que apoye a la pronta recuperación del agresor primario de la violencia de género y disminuir el feminicidio.

Las debilidades son las creencias sociales de machismo y de poder, a las cuales se aferra el sujeto para continuar con su valor de "ser hombre". La sociedad machista lo incita constantemente y por muchos medios, y el tratamiento debe ser un medio que promueva la igualdad social y de género.

\section{Conclusiones}

La investigación mixta cuantitativa y cualitativa nos brinda la oportunidad de triangular información analizando el fenómeno de agresión de género en voz de los agresores primarios de la pareja. Donde se observa el "qué" y su frecuencia y el "cómo" analizando el pensamiento y actitud ante el evento.

En voz del agresor podemos observar los disparadores contenidos en la alta frecuencia de concepción devaluada de la mujer, reflejado en el rechazo constante a las conductas positivas de la pareja $(84 \%)$. Las conductas de la pareja para obtener un valor y ejercer derechos le causan al agresor conflictos difíciles de resolver (72\%), porque el agresor está en la idea de conservar su lugar jerárquico de poder y protección social (física y mentalmente). Esta doble acción de querer y no poder le produce tristeza, apatía o rabia crónicas hacia la pareja, lo que provoca escaladas de agresión y/o abandono, distanciamiento emocional con la pareja (66 \%).

La lucha constante de lo aprendido y socialmente permitido, el agresor mantiene conductas de afrontamiento (75\%), con bajo control de impulsos (56\%), reforzadas por las malas ideas sobre la mujer, generadas por su comportamiento rebelde y agresivo. La intolerancia crea comportamientos desadaptados de escape manifestado en adicciones (53\%).

La no asunción de responsabilidad es recurrente (66\%), porque parte de la idea de que están ejerciendo su rol dominante y de protección ante la pareja porque la consideran de su propiedad. Y se encontró una frecuencia de tres a cuatro episodios violentos por semana (44\%); una manifestación de su agresividad por lapsos pequeños (minutos) (88\%), de intensidad leve (75\%) y con consecuencias igualmente leves $(63 \%)$. 


\section{Revista Iberoamericana \\ de las Ciencias Sociales y \\ Humanísticas}

ISSN: $2395-7972$

En esta última estadística se observa que el agresor solo desea mantener su rol social dominante y de protección de sus propiedades, para que su "ser valga", por eso sus agresiones las considera "leves", porque todo lo que hace está en función de conservar, controlar y proteger su relación de pareja. Cuando la pareja no quiere ser conservada, controlada y protegida se dispara el conflicto y las dimensiones y consecuencias este pueden llegar hasta el feminicidio, sin que el agresor se responsabilice, puesto que "así lo ha aprendido" y tiene que mantener su valor. Hay que recordar que estos participantes ya están diagnosticados con trastornos de relación humana, siguiendo el DSM-5 (APA, 2013).

El agresor primario requiere reaprender que la mujer no es de su propiedad y que tiene derechos para ejercer su propia vida. Por lo que el agresor requiere de justicia terapéutica para cambiar sus ideas y las formas de relacionarse con su pareja, y otorgarle el valor de ser independiente con capacidades de autodominio y fortaleza física y mental. 


\section{Revista Iberoamericana \\ de las Ciencias Sociales y \\ Humanísticas}

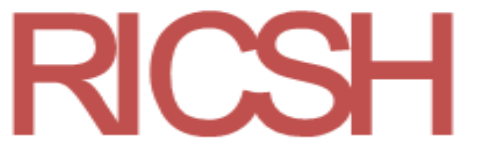

ISSN: $2395-7972$

\section{Referencias}

American Psychiatric Association [APA]. (2013). Diagnostic and Statistical Manual of Mental Disorders $\left(5^{\text {th }}\right.$ ed.). Arlington, United States: American Psychiatric Association.

Arce, R. y Fariña, F. (manuscrito no publicado). Entrevista semiestructurada para maltratadores de género.

Arce, R. y Fariña, F. (2010). Diseño e implementación del Programa Galicia de Reeducación de Maltratadores: Una respuesta psicosocial a una necesidad social y penitenciaria. Intervención Psicosocial, 19(2), 153-166. Recuperado de http://scielo.isciii.es/scielo.php?script=sci_arttext\&pid=S1132-05592010000200007.

Arce, R., Fariña, F. y Novo, M. (2014). Competencia cognitiva en penados primarios y reincidentes: implicaciones para la reeducación. Anales de Psicología, 30(1), 259-266. Recuperado de http://scielo.isciii.es/scielo.php?script=sci_arttext\&pid=S021297282014000100027.

Arce, R., Fariña, F., Vázquez, M. J., Novo, M. y Seijo, D. (2015). Programa Galicia de Reeducación de Agresores de Género. Santiago de Compostela, España: Andavira Editora.

Bejarano, M. (2014). El feminicidio es sólo la punta del iceberg. Religión y Sociedad, (4), 13-44. Recuperado de http://www.redalyc.org/articulo.oa?id=10230108002.

Castañeda, M. P., Ravelo, P. y Pérez, T. (2013). Feminicidio y violencia de género en México: omisiones del Estado y exigencia civil. Revista de Ciencias Sociales y Humanidades, 7(34), 11-39. Recuperado de https://www.redalyc.org/html/393/39348328002/.

Castillo, F. (2004) Género y educación en valores. Otra Mirada, 4(1), 20-29. Recuperado de http://redalyc.uaemex.mx/pdf/183/18340103.pdf.

Código Penal Federal. (12 de abril de 2019). Diario Oficial de la Federación. Recuperado de http://www.ordenjuridico.gob.mx/Documentos/Federal/pdf/wo83048.pdf.

Encuesta Nacional sobre la Dinámica de las Relaciones en los Hogares [Endireh]. (2016). Guía de autocuidado para tu seguridad emocional. México: Instituto Nacional de Estadística y Geografía. Recuperado http://internet.contenidos.inegi.org.mx/contenidos/productos/prod_serv/contenidos/espano 1/bvinegi/productos/nueva_estruc/promo/endireh2016_guia_autocuidado.pdf.

Espada, F. J. y Torres, P. (1996). Violencia en casa. Madrid, España: Aguilar.

Guerrera, F. (2018). Ni una más. El feminicidio en México un tema urgente en la Agenda Nacional. Ciudad de México, México: Editorial Aguilar. 


\section{Revista Iberoamericana \\ de las Ciencias Sociales y \\ Humanísticas}

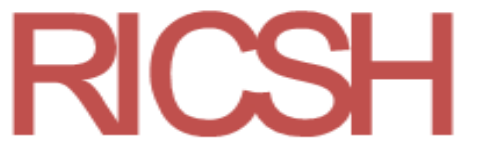

ISSN: $2395-7972$

H. Consejo de la Unión. (13 de abril del 2018). Ley General de Acceso de las Mujeres a una Vida Libre de Violencia. Nueva Ley. Diario Oficial de la Federación. Recuperado de http://www.diputados.gob.mx/LeyesBiblio/pdf/LGAMVLV_130418.pdf.

Instituto Nacional de Estadística y Geografía [Inegi]. (2016). Encuesta Nacional sobre la Dinámica de las Relaciones en los Hogares, 2016. Reporte Ejecutivo. México: Instituto Nacional de $\begin{array}{lllll}\text { Estadística } & \text { y } & \text { Geografía } & \text { Recuperado de }\end{array}$ https://www.inegi.org.mx/contenidos/programas/endireh/2016/doc/endireh2016_presenta cion_ejecutiva.pdf.

Instituto Nacional de Estadística y Geografía [Inegi]. (22 de noviembre de 2018). Estadísticas a propósito del Día Internacional de la Eliminación de la Violencia Contra la Mujer. Comunicado de Prensa Núm. 588/18 del Instituto Nacional de Estadística y Geografía. Recuperado de http://www.beta.inegi.org.mx/contenidos/saladeprensa/aproposito/2018/violencia2018_Na 1.pdf.

Jelev, J. (2006) Educación y ciudadanía. En Bindé, J. (dir.), ¿Hacia Dónde se Dirigen los Valores? Coloquios del siglo XXI (pp. 228-233). Ciudad de México, México: Fondo de Cultura Económica.

Klatt, P., Hahlweg, K., Job, A. and Foran, H. M. (unpublished manuscript). Cross-Validation of Screening Instruments for Intimate Relationship Distress.

Lila, M., Gracia, E. y Herrero, J. (2012). Asunción de responsabilidad en hombres maltratadores: influencia de la autoestima, la personalidad narcisista y la personalidad antisocial. Revista Latinoamericana de Psicología, 44(2), 99-108. Recuperado de http://www.fepsu.es/file/MALTRATADORES.pdf.

López, E. (2004). La figura del agresor en la violencia de género: características personales e intervención. Papeles del Psicólogo, 25(88), 31-38. Recuperado de http://www.redalyc.org/articulo.oa?id=77808805.

López, P. (2010). La violencia de género en el territorio latinoamericano, a través de la ocurrencia creciente de los feminicidios en la región. Revista Latinoamericana de Geografía de Género, Ponta Grossa, 1(1), 78-87.

Martínez, C. G. (3 de agosto de 2018). En México las mujeres no se sienten seguras. Noticias ONU. Recuperado de https://news.un.org/es/story/2018/08/1438852. 


\section{Revista Iberoamericana \\ de las Ciencias Sociales y \\ Humanísticas}

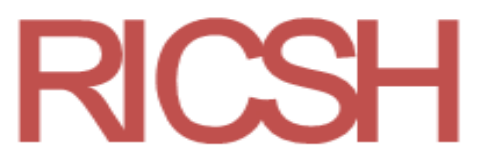

ISSN: $2395-7972$

Monárrez, S. (2009). Trama de una injusticia. Feminicidio sexual sistémico en Ciudad Juárez.

México: El Colegio de la Frontera y Porrúa.

Moscovici, S. (2002). Psicología Social. II Pensamiento y vida social. México: Paidós.

Observatorio Ciudadano Nacional del Feminicidio [OCNF]. (2014). Estudio de la implementación del tipo penal de feminicidio en México. Causas y consecuencias 2012 y 2013. México: Observatorio Ciudadano Nacional del Feminicidio.

Olamendi, P. (2016). Feminicidio en México. Ciudad de México, México: Gobierno de la República/Inmujeres.

Pérez, M, Giménez, A. y Espinosa, M. (2012). Evaluación del programa "Violencia de Género: programa de intervención para agresores", en medidas alternativas. Madrid, España: Secretaría General de Instituciones Penitenciarias, Ministerio del Interior y el Instituto de Ciencias Forenses y de la Seguridad de la Universidad Autónoma de Madrid. Recuperado de http://www.institucionpenitenciaria.es/web/export/sites/default/datos/descargables/des cargas/VDG_EVALUACION_AUTONOMA_NIPO.pdf.

Ramírez, K. y Echarri, C. (2010). Mapeo de procesos de atención y construcción de indicadores sobre casos de violencia contra las mujeres. México: Instituto Nacional de las Mujeres.

Rodríguez, N. P. (2011). Femicidio/Feminicidio: Una salida emergente de las mujeres frente a la violencia ejercida en contra de ellas. Revista Logos, Ciencia y Tecnología, 3(1) 127-148.

Romero, T. I. (2014). Sociología y política del feminicidio; algunas claves interpretativas a partir de un caso mexicano. Sociedad y Estado, 29(2), 375-391. Recuperado de http://www.scielo.br/scielo.php?script=sci_arttext\&pid=S0102-69922014000200004.

Russell, D. E. y Radford, J. (2006). Feminicidio. La política del asesinato de las mujeres. Ciudad de México, México: Universidad Nacional Autónoma de México.

Salvatierra, K. S. (2007). Reseña de "Feminicidio. La política del asesinato de las mujeres" de Diana E. Russell y Hill Radford. Revista Mexicana de Ciencias Políticas y Sociales, 49(200), 169-171. Recuperado de http://www.redalyc.org/pdf/421/42120010.pdf.

Segato, R. (2006). Qué es un feminicidio. Notas para un debate emergente. Brasilia, Brasil. Recuperado de https://www.nodo50.org/codoacodo/enero2010/segato.pdf.

Sociedad Mexicana De Psicología (2010). Código ético del psicólogo. México: Trillas.

Urbano, H. (2007). El enfoque etnometodológico en la investigación científica. Liberabit, 13(13), 89-91. Recuperado de http://www.redalyc.org/pdf/686/68601311.pdf. 
Revista Iberoamericana

de las Ciencias Sociales y

Humanísticas

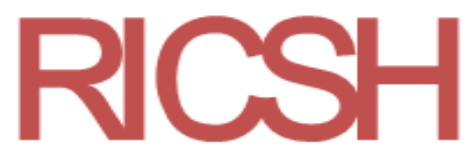

ISSN: $2395-7972$

Valle Sanpedro L. (2019). Nadine Gasman en el Inmujeres. EL Sol de México, análisis 5 marzo 2019. Recuperado de https://www.elsoldemexico.com.mx/analisis/nadine-gasman-en-elinmujeres-3143683.html

Whisman, M. A., Snyder, D. K. and Beach, S. R. H. (2009). Screening for marital and relationship discord. Journal of Family Psychology, 23, 247-254. 


\section{Revista Iberoamericana de las Ciencias Sociales y Humanísticas}

\begin{tabular}{|l|l|}
\hline Rol de Contribución & Autor (es) \\
\hline Conceptualización & Elvira Ivone González Jaimes \\
\hline Metodología & Elvira Ivone González Jaimes \\
\hline Software & NO APLICA \\
\hline Validación & Elvira Ivone González Jaimes \\
\hline Análisis Formal & María Guadalupe Miguel Silva \\
\hline Investigación & Elvira Ivone González Jaimes \\
\hline Recursos & Ana María Oviedo Zuñiga \\
\hline Curación de datos & María Guadalupe Miguel Silva \\
\hline $\begin{array}{l}\text { Escritura - Preparación del } \\
\text { borrador original }\end{array}$ & Elvira Ivone González Jaimes \\
\hline $\begin{array}{l}\text { Escritura - Revisión y } \\
\text { edición }\end{array}$ & Elvira Ivone González Jaimes \\
\hline Visualización & Ana María Oviedo Zuñiga. \\
\hline Supervisión & Ana María Oviedo Zuñiga \\
\hline Administración de Proyectos & Elvira Ivone González Jaimes \\
\hline Adquisición de fondos & Alejandro Mendieta Vargas \\
\hline
\end{tabular}

